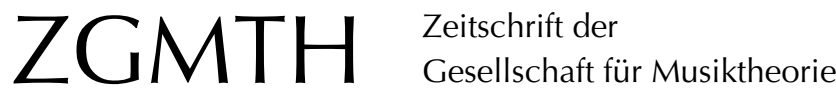

Stephan, Felix (2011): Reinhard Amon, Lexikon der Harmonielehre, Wien und München: Doblinger/Metzler 2005, und Lexikon der musikalischen Form, Wien und München: Doblinger/Metzler 2011. ZGMTH 8/3, 511-516. https://doi.org/10.31751/656 (C) 2011 Felix Stephan

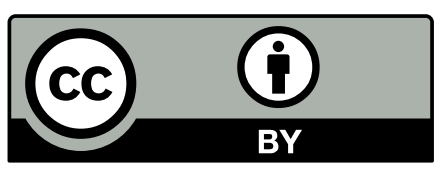

Dieser Text erscheint im Open Access und ist lizenziert unter einer Creative Commons Namensnennung 4.0 International Lizenz.

This is an open access article licensed under a Creative Commons Attribution 4.0 International License.

veröffentlicht / first published: 29/03/2012

zuletzt geändert / last updated: 09/11/2013 


\section{Reinhard Amon, Lexikon der Harmonielehre, Wien und München: Doblinger/Metzler 2005, und Lexikon der musikalischen Form, Wien und München: Doblinger/Metzler 2011}

»Kein Harmoniesystem ist in der Lage, alle harmonischen Erscheinungen hinreichend zu erfassen. $\ll^{1}$ - Diese vor nunmehr 20 Jahren vorgetragene Position Thomas Daniels darf mittlerweile als repräsentativ für den Mainstream gegenwärtiger musiktheoretischer Lehre und Forschung gelten.

Die ablehnende Haltung gegenüber dem universellen Geltungsanspruch musiktheoretischer Systeme hat ihren Ursprung in Carl Dahlhaus' Kritik an der Funktionstheorie, die er seit den späten 60er Jahren in unterschiedlichen Kontexten immer wieder thematisiert hat. $^{2}$ Dahlhaus forderte in erster Linie, die analytischen Werkzeuge den individuellen Eigenschaften des jeweiligen Werkes anzupassen. Er propagierte zu diesem Zweck einen »besonnenen Eklektizismus der theoretischen Ansätze $\aleph^{3}$ - mit weitreichenden Folgen. Statt eines "Kampfes der Systeme" dominieren heute, so Bernd Redmann, »die Auflösung der Systeme in einzelne Ideen oder Konstituenten und deren kritische Durchleuchtung sowie eine Tendenz zu systematischer und analytischer Methodenverknüpfung. ${ }^{4}$

Vor diesem Hintergrund erstaunt, dass vom jahrzehntelangen öffentlichen Diskurs nahezu unberührt - nach wie vor Lehrbücher erscheinen, in denen die Funktionstheorie als

1 Daniel 1992, 25. Weiter schreibt Daniel: »Weder die ,logische Funktionstheorie noch die spraktisches Stufentheorie bilden darin eine Ausnahme, von Rameaus basse fondamentale (Fundamentalbass) ganz zu schweigen.« (Ebd.)

2 Vgl. dazu exemplarisch Dahlhaus 1967.

3 Dahlhaus 1978, 215.

4 Redmann 2009, 59. universelles System der Musiktheorie inszeniert wird. ${ }^{5}$

In diese Reihe gehört auch Reinhard Amons Lexikon der Harmonielehre, das sich freilich dreifach von der breiten Masse aktuell verfügbarer Unterrichtsliteratur abhebt - hinsichtlich des Konzeptes, des Layouts und des Umfangs.

Amons Idee, Lexikon und Lehrbuch miteinander zu verknüpfen, verdient Respekt und könnte zur Nachahmung anregen. Die Vorteile liegen auf der Hand: Die lexikalische Ordnung von Fachbegriffen und Sachverhalten ermöglicht eine umfassende und gebündelte Gesamtdarstellung des Stoffes, während die lehrbuchartige Zusammenfassung zu größeren Themeneinheiten einerseits der Vertiefung und der Darstellung systematischer Zusammenhänge dient und andererseits Raum für Exkurse und die Thematisierung von Wissenswertem außerhalb des traditionellen 'Kanons` gibt.

Auch das Layout ist ansprechend. Durch farbige Bebilderungen und dreidimensionale Grafiken werden musiktheoretische Inhalte in lebendiger Weise veranschaulicht. Zahlreiche tabellarische Übersichten sorgen für zusätzliche Orientierung. Zwar ließe sich im Einzelfall darüber diskutieren, ob diese oder jene dreidimensionale Grafik wirklich zum Erkenntnisgewinn beiträgt (vgl. etwa die geradezu sschwindelerregender Darstellung eines "dreidimensionalen Quintenzirkels" in Beispiel 1). Doch im Großen und Ganzen hebt

$5 \mathrm{Zu}$ nennen sind in diesem Zusammenhang u. a. Thomas Krämers Lehrbuch der harmonischen Analyse (1997) und Doris Gellers Modulationslehre (2002). 
sich das Lexikon der Harmonielehre von der eher abweisenden Nüchternheit konkurrierender Lehrwerke wohltuend ab.

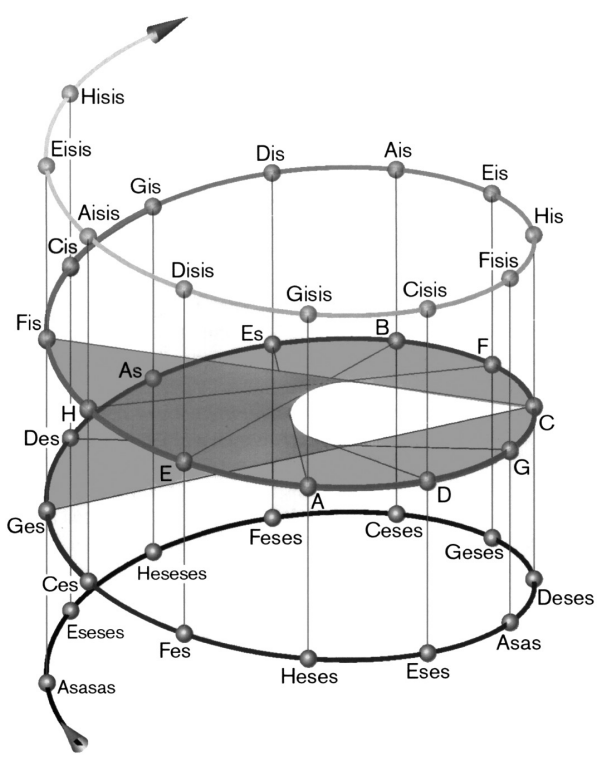

Beispiel 1: Reinhard Amon, Lexikon der Harmonielehre, Artikel „Quintenzirkel», Abschnitt »Dreidimensionaler Quintenzirkel«, S. 217

Der Umfang des Bandes ist mit über 400 kleingedruckten Seiten außergewöhnlich. Dies liegt nicht nur an der Ausführlichkeit, mit der Amon insbesondere die Funktionstheorie und ihre analytische Anwendung darstellt. Der Autor behandelt auch Themenbereiche, die über die Funktionstheorie zum Teil weit hinausgehen, und liefert beispielsweise Zusammenfassungen zur modalen Harmonik (S. 165-169), zu den verschiedenen Stimmungen (S. 253-262), zur Tonartencharakteristik (S. 288-293) und zu harmonischen Strukturen im 20. Jahrhundert (S. 365-381). Hier spiegelt sich die breit gefächerte Interessenlage des Wiener Tonsatzprofessors, der sich nicht nur als Musiktheoretiker, sondern auch als Klavierpädagoge einbringt. Eine Erweiterung des funktionstheoretischen Rahmens bedeutet auch die ergänzende Verwendung von Analysechiffren der Stufentheorie. Hier favorisiert der Autor die im englischsprachigen Raum übliche Groß- und Kleinschreibung der Stufen, wie sie z. B. in Robert Gauldins Standardwerk Harmonic Practice in Tonal Music zu finden ist.

Im Vorwort des Harmonielehre-Lexikons weist Amon darauf hin, dass der Leser »kein Werk mit neuen wissenschaftlichen Erkenntnissen« (S. 10) zu erwarten habe. Vielmehr gehe es ihm darum, bereits vorhandenes Wissen zusammenzutragen, neu zu ordnen und in zeitgemäßer Weise zu präsentieren.

Amons Hauptanliegen ist es, die Funktionstheorie als Instrument der harmonischen Analyse darzustellen. Eine überwältigende Vielzahl konstruierter Akkordfolgen, allesamt auf C-Dur bzw. c-Moll bezogen, wird durch eine relativ schmale Zahl authentischer Musikbeispiele ergänzt. Eine möglichst effektive und übersichtliche Darstellung scheint Amon wichtiger zu sein als die Exemplifikation der jeweils zur Rede stehenden Kategorien und Phänomene im kompositorischen Kontext. Besonders auffällig ist das weitgehende Fehlen von Literaturbeispielen bei der Darstellung von Modulationsvorgängen im Kapitel »Modulation« (S. 170-187). Der Wert der abstrakten Akkordfolgen, merkt Amon hierzu an, liege »im prinzipiellen Verstehen. Sie wollen und können Literaturbeispiele nicht ersetzen« (S. 173).

Auf einige "Schwächen der Funktionstheorie« kommt der Autor im Vorwort kurz zu sprechen (S. 9f.). So erwähnt er Schwierigkeiten der Funktionsanalyse im Umgang mit Sequenzen, mit modalen Satzstrukturen und mit Texturen, in denen von einer »Orientierung am Melodischen" auszugehen sei, die ein »Gegenprinzip« zur harmonischen Funktionalität darstelle. Auch die »Ableitung der Sixte ajouteé« wird problematisiert. Zwar hält der Autor in den meisten `Problemfällen` (wie etwa der II. Stufe in Moll) an den überkommenen funktionsharmonischen Erklärungsmustern fest, doch werden diese durch die Einbeziehung alternativer Nomenklaturen und Perspektiven ergänzt und relativiert. »Dort, wo die Funktionstheorie nicht greift, d. h. zur analytischen Klarheit beiträgt«, so der Autor, wer- 
de »auf Stufenchiffren umgestiegen, oder es werden Akkorde absolut bezeichnet« (S. 10).

Amon ist sich der Grenzen der Funktionstheorie also durchaus bewusst. Insbesondere dass er vielerorts auch historische Ansätze bei der Darstellung von musiktheoretischen Sachverhalten heranzieht, lässt sein Harmonielehre-Lexikon mehrdimensionaler erscheinen als vergleichbare Publikationen.

Bemerkenswert ist in dieser Hinsicht etwa der Artikel zu den »Klauseln« (S. 142 ff.). Hier werden - auch wenn die Darstellung teleologisch auf die smoderne Kadenzharmonikı hinzielt - wesentliche Zusammenhänge klar und detailliert dargestellt. So zeigt Amon unter anderem, dass sowohl der Quartvorhalt auf der Dominante als auch die 'Sixte ajoutée` aus alternativen Bassunterlegungen des Tenor-DiskantGerüsts hervorgehen können (S. 144 oben).

Erfreulich ist auch der Umstand, dass Amon in einem separaten Kapitel die verschiedenen »Theorien« von ihren systematischen Grundlagen und ihrem jeweiligen historischen Kontext her zur Darstellung bringt - von der Generalbasslehre über die Fundamentalbassund die Stufentheorie bis hin zu den verschiedenen Spielarten der Funktionstheorie einschließlich des harmonischen Dualismus (S. 264-273). Auch `Tonalitäten jenseits der Dur-Moll-Tonalität und den Mitteln der »Tonalitätsauflösung« und »Tonalitätserweiterung" wird Raum gegeben, wobei allerdings unklar bleibt, welchem übergeordneten Begriff von `Tonalität der Autor folgt (S. 274-287).
Es lässt sich viel Gelungenes in diesem Buch finden, gerade auch dort, wo Amon über die bloße Harmonielehre hinausgeht. Sein Betrag zur Tonartencharakteristik beispielsweise (S. 288-293) fasst übersichtlich und griffig zusammen, was man sich sonst mühsam aus verschiedenen Quellen zusammensuchen müsste.

Allerdings bergen kleinere Unschärfen, Verkürzungen oder Verallgemeinerungen, die aufs Ganze gesehen kaum ins Gewicht fallen, die Gefahr, bei weniger kundigen Lesern zu Missverständnissen zu führen.

So behauptet Amon beispielsweise im Kapitel "Harmonischer Rhythmus", in der Wiener Klassik habe sich die Geschwindigkeit der Tempi »verdoppelt» (S. 103). Er beruft sich hierbei auf Dieter de la Mottes Harmonielehre (S. $136 \mathrm{ff}$.), in der es jedoch lediglich heißt, die relative Verlangsamung des harmonischen Aktionstempos habe in der Klassik ein neues Tempo ermöglicht, nämlich das Presto.

Wo Amon Literaturbeispiele bringt, dienen sie stets der Darstellung bestimmter musiktheoretischer Kategorien oder Sachverhalte. So führt er die ersten vier Takte von Beethovens »Waldsteinsonate« op. 57 als Beispiel für eine »Rückung» an (Beispiel 2).

Dies ist in mehrfacher Hinsicht problematisch: Dass es sich bei der von Amon diskutierten Akkordfolge $\mathrm{C}-\mathrm{G}_{3} / \mathrm{B}-\mathrm{F}_{3}$ um eine quasi ssequenzielle، Versetzung handelt, bleibt unerwähnt; auch fehlt das sarchaische`Modell (Quintstieg, sekundweise fallend) in Amons

L. v. BeEthoven: Klaviersonate C-Dur op. 53, "Waldsteinsonate", 1. Satz, Beginn - Reduktion auf das harmonische Gerüst

Die weiterführenden, auf die Ausgangstonart C-Dur bezogenen Funktions- und Stufenchiffren (hellgrau) sind möglich, werden aber dem Sachverhalt der »Rückung« nicht gerecht. Zwischen G-Dur (dritter Akkord) und B-Dur (vierter Akkord) erfüllt der Ton d als Tonzentrierung die Funktion einer klanglichen Verbindung. Die ersten drei Akkorde sind als S-D-T von G-Dur interpretierbar, die beiden letzten Akkorde als D-T von F-Dur.

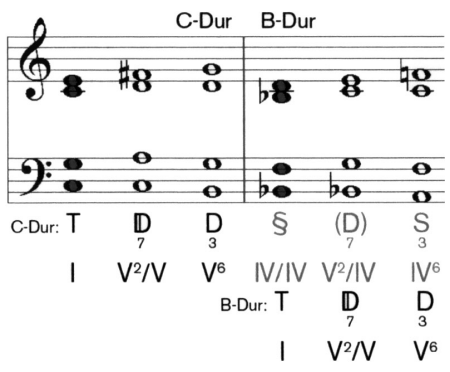

Beispiel 2: Reinhard Amon, Lexikon der Harmonielehre, Artikel »Modulation«, Abschnitt »Rückung - Tonalitätssprung« S. 173 
Sequenzübersicht (S. 238). Dabei ist es gerade die smechanische Fortschreitungslogik der sekundweisen Versetzung, die den harmonischen Schnitt erst hervorbringt und zugleich slegitimiert. Überdies sind die ersten vier Takte Teil einer modellhaft-kontrapunktischen Formulierung des absteigenden chromatisierten Tetrachords (T. 1-5), das vom tonikalen $\mathrm{C}$ in das dominantische G führt. Der Zwang, um der Reduktion auf das für die Kategorie der ^Rückung، Wesentliche willen, den tonalen Zusammenhang auszublenden und stattdessen eine Art >Ameisenperspektiver einzunehmen, spiegelt sich auch in Amons funktionsharmonischer Interpretation des BDur-Akkords als lokale sTonikar. Unmittelbar verständlich hingegen wird der B-Dur-Akkord an dieser Stelle als subdominantische Antepenultima eines auf die globale Subdominante gerichteten Kadenzvorgangs. Dass Amon im Rahmen eines knappen Lexikonartikels nicht alle wesentlichen Aspekte eines Literaturbeispiels beleuchtet, ist ihm nicht vorzuwerfen. Zum Problem wird die Wahl eines Beispiels jedoch dann, wenn das jeweilige Darstellungsinteresse zu Deutungen zwingt, die den Gegenstand offenkundig verfehlen.

Schließlich mag man es bedauern, auch bei einem in vieler Hinsicht sehr differenziert argumentierenden Autor gängige, durch den Fachdiskurs überholte (Vor-)Urteile unhinterfragt fortgeschrieben zu sehen. So konstantiert Amon beispielsweise, die Bedeutung des Generalbasses als theoretisches System sei gering, da er »nichts über die Bedeutung von Akkordtönen und Akkorden selbst oder die Beziehungen der Akkorde zueinander « aussage (S. 264). Forschungsergebnisse, die über die begrenzte Sicht des Generalbasses als eine bloße >Chiffriermethode` weit hinausweisen, bleiben unberücksichtigt. Amon zitiert in diesem Zusammenhang Hugo Riemann, der den Generalbass "schmählich als Vehikel der Harmonielehre bezeichnet« habe. Wo in den Schriften Riemanns dieses Zitat zu finden ist, teilt er nicht mit. Tatsächlich verwendet Riemann in seiner Geschichte der Musiktheorie eine ähnliche Formulierung. ${ }^{6}$ Dort spricht er vom Generalbass als einem »Vehikel der Satz- lehre«, setzt jedoch hinzu, dieser habe »dem Wachsen der theoretischen Erkenntnis große und unschätzbare Dienste geleistet«.

Sechs Jahre nach Erscheinen des Lexikons der Harmonielehre hat Amon ein zweites Theorie-Lehrwerk veröffentlicht - das Lexikon der musikalischen Form.

Noch deutlicher als in seinem Harmonielehre-Lexikon formuliert Amon hier ästhetische Standpunkte, die erkennbar im 19. Jahrhundert wurzeln: "Der Wunsch und die Freude, sich mit Musik als Form zu befassen, entspringt der Faszination, die von ihrer Schönheit ausgeht", schreibt Amon zu Beginn des Vorworts (S. 8). "Musikalische Schönheit", so die Definition des Autors, sei die "geschaffene Einheit von Klang und Form, Material und Gestalt."

Das bewährte Konzept des Harmonielehre-Lexikons - die Kombination aus Lehrbuch und Lexikon - hat Amon im Lexikon der musikalischen Form noch erweitert. Neben einem umfangreichen Lexikonteil gibt es nun zwanzig zusammenfassende Beiträge, die durch zahlreiche klug durchdachte Verweise mit dem Lexikonteil verknüpft sind. Amon vertieft in diesen Kapiteln nicht nur Prinzipien der musikalischen Formung, sondern stellt auch interdisziplinäre Verbindungen zur Architektur, zur Wahrnehmungspsychologie, zur Philosophie und zur Mathematik her.

Ähnlich wie das Lexikon der Harmonielehre bietet also auch das Lexikon der musikalischen Form sehr viel mehr, als man es von einem herkömmlichen Musiktheorie-Lehrwerk gewohnt ist. Dass Amon darüber hinaus auch den wichtigsten populären Strömungen des 20. Jahrhunderts - Jazz, Rock und Pop - Raum gibt, ist ein Novum im deutschsprachigen Raum und dem Autor hoch anzurechnen. Ungeachtet aller Knappheit werden diese wesentlichen Stilbereiche der U-Musik kompetent und unter Berücksichtigung zentraler Aspekte und Begriffe zur Darstellung gebracht

6 Riemann 1921, 435. 
ELTON John, Your Song, T. 3-6

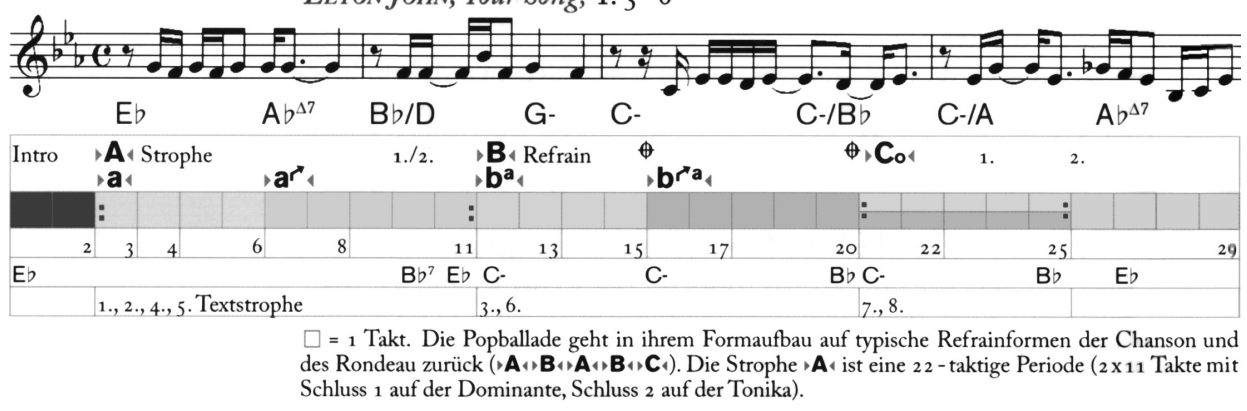

Beispiel 3: Reinhard Amon, Lexikon der musikalischen Form, Kap. »Formstrukturen in Popularmusik und Jazz", Beispiel für einen 'Verse` (obere Notenzeile) sowie für den Formaufbau einer Popballade (Analysegrafik) S. 476

(vgl. dazu exemplarisch die Formanalyse von Elton Johns Your Song in Beispiel 3).

Im Bereich der traditionellen Formenlehre setzt Amon klare Prioritäten. So gewährt er im Bereich barocker Formen der 'Fuge 16 Seiten den größten Raum, die 'Triosonates dagegen wird auf lediglich einer Seite behandelt. Im klassischen Bereich beansprucht die 'Sonatensatzform 13 Seiten, während für das 'Menuett - in einem shistorisch informierten Tonsatzunterricht die zentrale klassische Form - nur zwei Seiten bleiben. Diese Gewichtung ist dadurch zu erklären, dass Amon das Lexikon der musikalischen Form, ähnlich wie bereits das Lexikon der Harmonielehre, primär mit Blick auf die musikalische Analyse konzipiert hat.

Im Vorwort schreibt Amon, er habe sich bewusst dagegen entschieden, den Begriff „Formenlehre« im Buchtitel zu führen. Denn diese Bezeichnung berge die Gefahr, musikalische Form als »oberflächlichen Schematismus « misszuverstehen und verstelle den Blick auf die lebendige, klingende Musik (S. 8).

Nähme man den Autor beim Wort, so wäre es nur konsequent, ebenso den Begriff 'Harmonielehre zu hinterfragen. Denn auch die 'Harmonielehre beschäftigt sich mit lebendiger Musik, und auch hier besteht die Gefahr eines oberflächlichen Schematismus, insbesondere wenn vermeintlich suniverselle، Theoriesysteme verwendet werden.
Anders als im Harmonielehre-Lexikon zieht Amon im Lexikon der musikalischen Form eine Fülle von insgesamt über 600 Werkausschnitten als Anschauungsmaterial heran. Das "Konzept der Analyse mittels Tabellen, Farben und Symbolen« (S. 10) entwickelte Amon gemeinsam mit dem Musikwissenschaftler Gerold Gruber, der auch als Co-Autor genannt wird. Erfreulicherweise wählen Amon und Gruber überwiegend relativ sunverbrauchtes und dennoch leicht greifbare Beispiele. Dies macht das Lehrbuch besonders attraktiv für Lehrende, die sich die eigenständige Suche nach geeignetem Anschauungsmaterial ersparen möchten.

Allerdings gibt es einige Fälle, in denen die Literaturbeispiele nicht optimal gewählt sind oder ihre jeweilige Deutung diskussionswürdig wäre. So führt Amon als Beispiel für »Anhang" bzw. "äußere Erweiterung" das Seitenthema aus dem 1. Satz der h-Moll-Sinfonie von Franz Schubert (T. 44-51) an. Seiner Ansicht nach bilden die Takte 50f. hier eine äußere Erweiterung (S. 25). Problematisch erscheint diese Deutung insofern, als Amon ausschließlich auf die Taktgruppenstruktur abhebt, den verklammernden harmonischen Zusammenhang jedoch unbeachtet lässt.

Seine Ankündigung, auch »historischen Sichtweise[n] " gerecht werden zu wollen (S. 9), löst Amon zumindest teilweise ein. So thematisiert er ausführlich die 'Klangredes 
Johann Matthesons (S. 182-184). Die für das Verständnis der Musik des 18. Jahrhunderts eher noch wichtigere Interpunktionslehre Heinrich Christoph Kochs dagegen wird nur am Rande erwähnt und bleibt in Amons Analysen ohne Reflex.

Leider resultieren aus Amons Tendenz zu einer extrem verknappten Darstellung gelegentlich auch missverständliche Formulierungen. So definiert Amon beispielsweise die Quintfallsequenz als »in fallenden Quintschritten aufeinander-folgende Dreiklänge $[\ldots]$, die [...] kurzfristige Dominant-TonikaVerhältnisse eingehen.« (S. 293) Abgesehen davon, dass an der Quintfallsequenz zumeist (auch) Vierklänge beteiligt sind, kann im Regelfall der diatonischen Quintfallsequenz von »kurzfristige[n] Dominant-TonikaVerhältnisse[n] « allenfalls aus Sicht der ramistischen Fundamentschritttheorie (`Dominantenkette $)$, nicht jedoch im Sinne der Funktionstheorie die Rede sein. Auch unterbleibt jeder Hinweis auf die Linearität des Oberstimmensatzes oder die historische Entwicklung der Quintfallsequenz aus dem Unterquintkanon und der 7-6-Konsekutive - analytisch relevante Aspekte, die im Lexikon der Harmonielehre zumindest ansatzweise thematisiert wurden (S. $235 \mathrm{ff}$.).

\section{Fazit}

Der Umfang beider Lexika von insgesamt 1055 Seiten ist ebenso beeindruckend wie ihre thematische Breite und ihre klare, leserfreundliche Gesamtanlage. Gleichwohl stellt sich ganz grundsätzlich die Frage, ob enzyk- lopädische Gesamtdarstellungen, die - ungeachtet innovativer Präsentationsformen - im Kern nach wie vor den traditionellen Kategorien und Systematiken der musiktheoretischen Teildisziplinen 'Harmonielehre und 'Formenlehre verpflichtet sind, noch als zeitgemäß gelten können: Trotz des Bemühens um die Integration sowohl 'historischer Perspektiven als auch von Gegenständen und Fragestellungen, die in herkömmlichen Harmonie- und Formenlehren keine Berücksichtigung finden, bleiben beide Bände in mancher Hinsicht hinter dem Stand des gegenwärtigen Fachdiskurses im deutschsprachigen Raum zurück.

Andererseits gibt es derzeit keine anderen deutschsprachigen Publikationen, die ein so breites Überblickswissen in vergleichbar gebündelter und vertiefter Form verfügbar machen: Die große Informationsdichte, die inspirierende Aufbereitung von musikalischen Lehrinhalten und die interdisziplinären Exkurse setzen auf dem deutschsprachigen Lehrbuchmarkt neue Maßstäbe. Vor diesem Hintergrund wird man die Bände auch Schülern und Studierenden empfehlen können - trotz kleinerer Unschärfen, einzelner ungünstig gewählter Beispiele und gelegentlich einseitiger Akzentuierungen. Umso mehr wird der kundige, einer gegebenenfalls auch kritischen Lektüre fähige Leser beide Bände immer wieder mit Gewinn konsultieren, da sie vielfach die Suche nach geeigneten Beispielen zur Darstellung bestimmter Sachverhalte ersparen und übersichtliche Zusammenfassungen sowie knappe Definitionen an die Hand geben.

Felix Stephan

\section{Literatur}

Dahlhaus, Carl (1967), Untersuchungen über die Entstehung der harmonischen Tonalität, Kassel: Bärenreiter.

Dahlhaus, Carl (1978), »Tristanı-Harmonik und Tonalität", Melos 45, 215-219.

Daniel, Thomas (1992), Der Choralsatz bei Bach und seinen Zeitgenossen, Köln: Dohr.
Redmann, Bernd (2009), »Funktionstheorie«, in: Systeme der Musiktheorie, hg. von Clemens Kühn und John Leigh, Dresden: Sandstein, 56-69.

Riemann, Hugo (1921), Geschichte der Musiktheorie im 9.-19. Jahrhundert, 2. Auflage, Berlin: Hesse. 